\title{
HOXA4 protein levels and localization in the aorta and in human abdominal aortic aneurysms
}

\author{
Christian Klausen ${ }^{*}$ and Nelly Auersperg
}

\begin{abstract}
This report presents evidence for the specificities of select commercially available HOXA4 antibodies in regards to concerns about the specificity of the HOXA4 antibody used by Lillvis et al. (Regional expression of HOXA4 along the aorta and its potential role in human abdominal aortic aneurysms. BMC Physiol 2011, 11:9). Using an antibody characterized extensively by us, Lillvis et al. report detecting HOXA4 at a size of $33 \mathrm{kDa}$ despite our previous reports that HOXA4 is detected at $\sim 37-39 \mathrm{kDa}$ and that the $\sim 30-33 \mathrm{kDa}$ band is non-specific. Using small interfering RNA targeting HOXA4, forced expression of full-length HOXA4 and HOXA4-positive and -negative ovarian cancer cell lines, we confirm our previous findings that the $\sim 30-33 \mathrm{kDa}$ band is non-specific and that HOXA4 is detected at $\sim 37-39 \mathrm{kDa}$. Moreover, we demonstrate that HOXA4 small interfering RNA reduces the 37$39 \mathrm{kDa}$ HOXA4 band, but not the $30-33 \mathrm{kDa}$ non-specific band, in a human acute monocytic leukemia cell line used by Lillvis et al. Western blot analysis performed with two additional commercially available HOXA4 antibodies also detected HOXA4 at 37-39 kDa. Lastly, immunofluorescent staining of a HOXA4-negative ovarian cancer cell line with the antibody used by Lillvis et al. yields strong perinuclear staining, similar to that observed by Lillvis et al., which cannot be attributed to HOXA4. Our results highlight and briefly discuss the importance of careful antibody validation and selection for use in various applications.
\end{abstract}

\section{Correspondence}

We read with interest the study of Lillvis et al. [1] regarding the expression of HOXA4 in the aorta and its potential role in abdominal aortic aneurysms. The authors used microarray analysis validated by reverse transcription quantitative real-time PCR to provide strong evidence that HOXA4 mRNA levels are reduced in human abdominal aortic aneurysms relative to control human abdominal aorta. However, we have significant concerns about the subsequent data regarding HOXA4 protein levels. For their studies Lillvis et al. used a commercially available rabbit polyclonal HOXA4 antibody (ab26097; Abcam, Cambridge, MA) that was previously characterized extensively by us $[2,3]$. While they were kind enough to reference our studies, they state that HOXA4 was detected as a single band at $\sim 33$ $\mathrm{kDa}$ and evidence for this is presented in their Additional file three, Figure S1A. However, in both of our previous studies we state that the size of HOXA4 is

\footnotetext{
* Correspondence: klausen@mail.ubc.ca

Department of Obstetrics \& Gynaecology, Child \& Family Research Institute,
} University of British Columbia, Vancouver, British Columbia, Canada
37-39 $\mathrm{kDa}$ and our first study [2] demonstrated that the band at $\sim 30-33 \mathrm{kDa}$ is a non-specific band.

The $\sim 30-33 \mathrm{kDa}$ non-specific band is by far the most intense band and appears as a single band at low exposures irrespective of blotting conditions, as was observed by Lillvis et al. in Additional file three, Figure S1A. This was demonstrated previously by us [2] and we now provide additional evidence of this in Figures $1 \mathrm{~A}$ and $1 \mathrm{~B}$. Strong expression of the $\sim 30-33 \mathrm{kDa}$ non-specific band is observed in the five human ovarian cancer cell lines shown in Figures $1 \mathrm{~A}$ and $1 \mathrm{~B}$ regardless of the fact that HOXA4 mRNA is undetectable in both SKOV-3 and A2780 cells $[2,3]$. More importantly, the $\sim 30-33 \mathrm{kDa}$ non-specific band is insensitive to small interfering RNA (siRNA) targeting HOXA4 ([2] and Figure 1A) and forced expression of full-length HOXA4 (Figure 1B). Likewise, Figure 1A shows strong, siRNA-insensitive expression of the $\sim 30-33 \mathrm{kDa}$ non-specific band in the acute monocytic leukemia cell line used by Lillvis et al. (THP-1 cells, designated as MP1 cells by Lillvis et al. in Additional file four, Table S3).

In contrast, we have demonstrated that the $\sim 37-39$ kDa HOXA4 band correlates with HOXA4 mRNA 


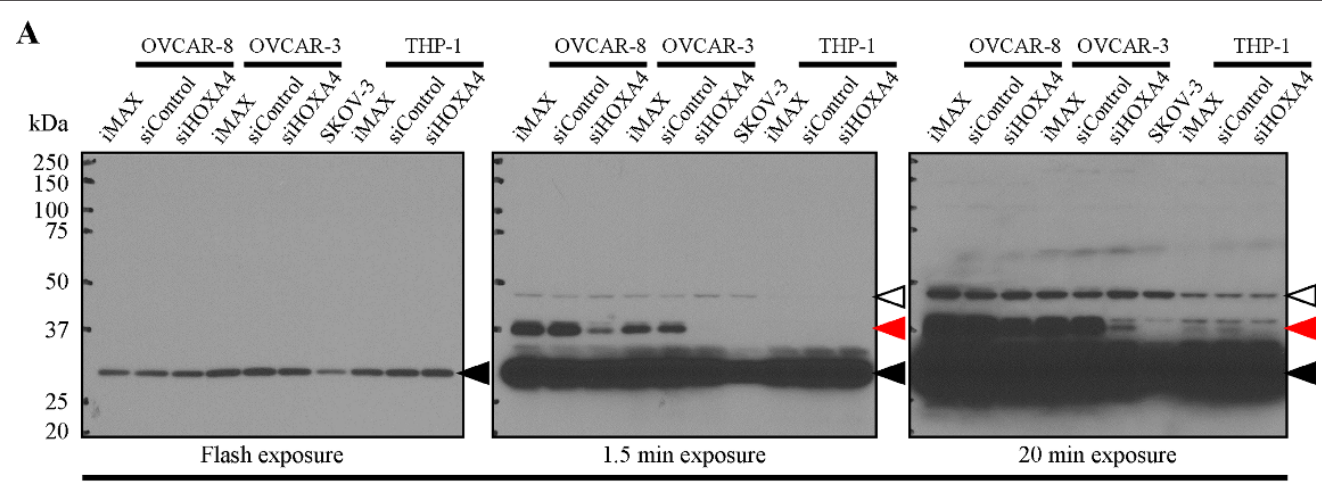

Rabbit polyclonal HOXA4 (Abcam ab26097) 1:40000

B

$(0.0125 \mu \mathrm{g} / \mathrm{mL})$

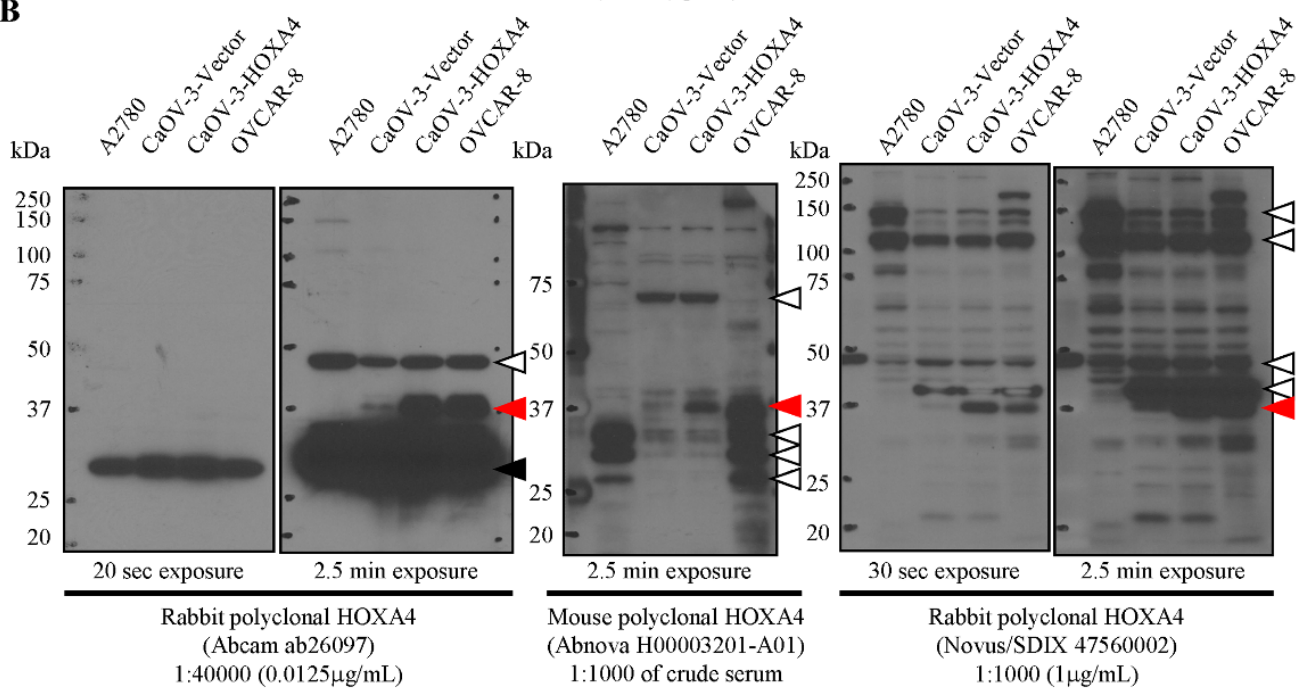

C

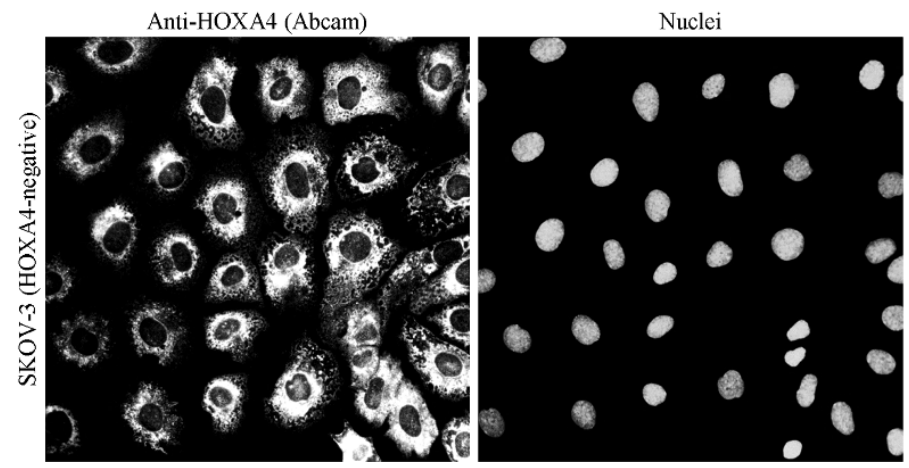

Figure 1 Evidence for the specificities of select commercially available HOXA4 antibodies. Immunoblot analysis was performed as in [3] with lysates from one human acute monocytic leukemia (THP-1) and five ovarian cancer (OVCAR-8, OVCAR-3, SKOV-3, A2780 and CaOV-3) cell lines. Red arrowheads indicate HOXA4 ( 37-39 kDa), black arrowheads indicate the $\sim 30-33 \mathrm{kDa}$ non-specific band associated with the Abcam antibody, and white arrowheads indicate select non-specific bands of equal/greater intensity. (A) SKOV-3 cells lack HOXA4 whereas OVCAR-8 and OVCAR-3 cells express high levels of HOXA4 [2,3]. Transient knockdown was performed as in [3] with 20 nM HOXA4-targeting siRNA (siHOXA4; ON-TARGETplus SMARTpool; Dharmacon), control siRNA (siControl; ON-TARGETplus Non-Targeting Pool) or transfection reagent alone (iMAX; Lipofectamine RNAiMAX). Immunoblot analysis with the Abcam HOXA4 antibody characterized by us [2,3] shows that the $~ 30-33$ kDa nonspecific band detected by Lillvis et al. [1] is insensitive to HOXA4 siRNA and is expressed by HOXA4-negative SKOV-3 cells. (B) A2780 cells lack HOXA4 whereas CaOV-3 cells express low levels of HOXA4 [2,3]. CaOV-3 cells were transfected as in [3] with control vector (CaOV-3-Vector) or vector encoding full-length HOXA4 (CaOV-3-HOXA4). Immunoblot analysis with the Abcam antibody and two other commercially available HOXA4 antibodies shows that the 37-39 kDa HOXA4 band detects exogenously expressed HOXA4 and is undetectable in HOXA4-negative A2780 cells. (C) Formaldehyde-fixed SKOV-3 cells were permeabilized, blocked and incubated overnight with anti-HOXA4 (1:3200). Secondary antibody was applied, cell nuclei were stained with Hoechst 33258, and coverslips were examined by epifluorescence microscopy. Although HOXA4 mRNA [2,3] and protein (A) are undetectable in SKOV-3 cells, these cells have strong expression of the 30-33 kDa non-specific band ((A) and [2]). Note that immunofluorescent staining of HOXA4-negative SKOV-3 cells with the Abcam antibody yields strong perinuclear staining which cannot be attributed to HOXA4. 
levels, is sensitive to HOXA4 siRNA and detects exogenously expressed HOXA4 ([2,3] and Figures $1 \mathrm{~A}$ and 1B). Although the $\sim 37-39 \mathrm{kDa}$ HOXA4 band is expressed at very low levels in THP-1 cells, it is the only band that is reduced by treatment with HOXA4 siRNA (Figure 1A). Furthermore, we now provide evidence that two additional commercially available HOXA4 antibodies detect a $\sim 37-39 \mathrm{kDa}$ band that correlates with HOXA4 mRNA levels and detects exogenously expressed HOXA4 (Figure 1B). In this context, we are not convinced that the Western blot results presented by Lillvis et al. reflect changes in HOXA4 protein levels. In light of their convincing mRNA data, perhaps a re-examination of the results of longer exposures would allow Lillvis et al. to quantify the $\sim 37-39 \mathrm{kDa}$ HOXA4 band detected with this antibody. We routinely cut away the membrane just below the $37 \mathrm{kDa}$ molecular mass marker prior to immunoblotting in order to prevent strong signal from the $\sim 30-33 \mathrm{kDa}$ non-specific band from interfering with quantitation of the $\sim 37-39$ kDa HOXA4 band.

Our second, and very much related, concern has to do with the use of the same rabbit polyclonal HOXA4 antibody for immunohistochemistry and immunofluorescence. In light of the intense $\sim 30-33 \mathrm{kDa}$ non-specific band and additional $\sim 46-48 \mathrm{kDa}$ non-specific bands of equal or greater intensity than HOXA4, we have significant concerns about the use of this antibody in these types of applications. Indeed, we have performed immunofluorescent staining of HOXA4-negative SKOV-3 cells $([2,3]$ and Figure 1A) and found strong perinuclear staining similar to that observed by Lillvis et al. which cannot be attributed to HOXA4 since SKOV-3 cells don't express HOXA4 (Figure 1C). While this staining most likely corresponds to the $\sim 30-33 \mathrm{kDa}$ non-specific band due to its intensity and the high dilution of the antibody (1:3200 vs. 1:200 used by Lillvis et al.), we cannot rule out contributions from the $\sim 46-48 \mathrm{kDa}$ nonspecific bands. Nuclear localization, especially as assessed by epifluorescence rather than confocal microscopy, does not appear to be sufficient to identify HOXA4 in cells with demonstrated HOXA4 expression since Lillvis et al. also report detecting the $\sim 30-33 \mathrm{kDa}$ band in both cytoplasmic and nuclear fractions by Western blot.

In this context, we are not convinced that the immunohistochemistry and immunofluorescence results presented by Lillvis et al. accurately depict the localization of HOXA4 protein. Indeed, we would suggest that the use of this rabbit polyclonal HOXA4 antibody in these types of applications is unwarranted unless definitive validation of specificity is presented. Our preliminary Western blot analysis of two additional commercially available HOXA4 antibodies has similarly revealed a number of non-specific bands of equal or greater intensity than HOXA4 (Figure 1B). Persuasive validation studies will therefore be required to establish whether any of the commercially available HOXA4 antibodies possess the specificity required for use in applications such as immunohistochemistry and immunofluorescence. Future studies on the biology of HOXA4 and its roles in disease would benefit greatly from the development of a highly specific antibody suitable for use in these and other applications.

The example of HOXA4 highlights the need for researchers to validate the specificity of antibodies for use in various applications, especially in the absence of rigorous antibody characterization. Indeed, while the Abcam HOXA4 antibody performs very well in Western blot analysis, its use in other applications such as immunocytochemistry, immunohistochemistry or immunoprecipitation is likely unwarranted unless definitive validation studies are performed in consideration of the sheer intensity of the signal from the $\sim 30-33 \mathrm{kDa}$ protein. For many companies antibody characterization takes the form of ELISA or Western blot analysis with the antigenic peptide/protein. While this does establish whether or not the antibody can detect the particular peptide/protein against which it was raised, it does not prove it will detect the actual protein (except when raised and tested against full-length protein) nor does it address how the antibody will perform in the context of the entire proteome (i.e. in cells or tissues) or in different applications. Preabsorption studies with the antigenic peptide/protein can identify true non-specific bands (i.e. cannot be eliminated by saturation), but they cannot distinguish the protein of interest from non-specific proteins with similar epitopes that the antibody binds to in a specific manner. For example, preabsorption of the Abcam HOXA4 antibody with the antigenic peptide completely eliminates the $\sim 37-39$ kDa HOXA4 band, the $\sim 30-33 \mathrm{kDa}$ non-specific band and one of the $\sim 46-48 \mathrm{kDa}$ non-specific bands [2]. Thus, the other $\sim 46-48 \mathrm{kDa}$ non-specific band is likely a true non-specific band, yet this approach fails to identify only the $\sim 37$ $39 \mathrm{kDa}$ band as being specific for HOXA4.

The detection of a band of "appropriate" size in cell or tissue lysates can be used to confirm the identity of a band, but must involve more than one (preferably three or more) lysates from cells or tissues known to have differing basal or experimentally manipulated expression levels. In general, accurate up-front knowledge of expression levels is most easily achieved by measuring mRNA levels. An obvious, and highly effective, extension of this approach is to use RNA interferencemediated knockdown or vector-based forced expression to generate lysates with gene-specific changes in the expression level of the protein of interest. Although use 
of an antibody in several applications requires separate validation for each application, many of the approaches discussed above can be adapted to different applications. In addition, companies are beginning to employ these approaches as they strive to market gene-specific product portfolios (antibody, protein/peptide, RNA interference and forced expression) on a genome-wide scale. However, in the absence of thorough antibody characterization it is ultimately the responsibility of the researcher to ensure the validity of results obtained from antibody-based applications.

\section{Acknowledgements}

This work was supported by a grant from the National Cancer Institute of Canada with funds from the Canadian Cancer Society to N.A.

\section{Authors' contributions}

CK conceived of the study, participated in its design, carried out all experiments and drafted the manuscript. NA participated in the design of the study and helped to draft the manuscript. All authors read and approved the final manuscript.

\section{Competing interests}

The authors declare that they have no competing interests.

Received: 22 August 2011 Accepted: 14 December 2011

Published: 14 December 2011

\section{References}

1. Lillvis JH, Erdman R, Schworer CM, Golden A, Derr K, Gatalica Z, Cox LA, Shen J, Vander Heide RS, Lenk GM, Hlavaty L, Li L, Elmore JR, Franklin DP, Gray JL, Garvin RP, Carey DJ, Lancaster WD, Tromp G, Kuivaniemi H: Regional expression of HOXA4 along the aorta and its potential role in human abdominal aortic aneurysms. BMC Physiol 2011, 11:9.

2. Ota T, Klausen C, Salamanca MC, Woo HL, Leung PC, Auersperg N: Expression and function of HOXA genes in normal and neoplastic ovarian epithelial cells. Differentiation 2009, 77:162-171.

3. Klausen C, Leung PC, Auersperg N: Cell motility and spreading are suppressed by HOXA4 in ovarian cancer cells: possible involvement of beta1 integrin. Mol Cancer Res 2009, 7:1425-1437.

doi:10.1186/1472-6793-11-18

Cite this article as: Klausen and Auersperg: HOXA4 protein levels and localization in the aorta and in human abdominal aortic aneurysms. BMC Physiology 2011 11:18.

\section{Submit your next manuscript to BioMed Central and take full advantage of:}

- Convenient online submission

- Thorough peer review

- No space constraints or color figure charges

- Immediate publication on acceptance

- Inclusion in PubMed, CAS, Scopus and Google Scholar

- Research which is freely available for redistribution

Submit your manuscript at www.biomedcentral.com/submit
C Biomed Central 